\title{
Geometry and General Relativity in the Groupoid Model with a Finite Structure Group
}

\author{
Michael Heller \\ Copernicus Center for Interdisciplinary Studies, Cracow, Poland \\ Tomasz Miller* Leszek Pysiak Wiesław Sasin \\ Faculty of Mathematics and Information Science, \\ Warsaw University of Technology \\ ul. Koszykowa 75, 00-662 Warsaw, Poland \\ and Copernicus Center for Interdisciplinary Studies, \\ Cracow, Poland
}

October 8, 2018

\begin{abstract}
In a series of papers [1, 2, 3] we proposed a model unifying general relativity and quantum mechanics. The idea was to deduce both general relativity and quantum mechanics from a noncommutative algebra $\mathcal{A}_{\Gamma}$ defined on a transformation groupoid $\Gamma$ determined by the action of the Lorentz group on the frame bundle $\left(E, \pi_{M}, M\right)$ over space-time $M$. In the present work, we construct a simplified version of the gravitational sector of this model in which the Lorentz group is replaced by a finite group $G$ and the frame bundle is trivial $E=M \times G$. The model is fully computable. We define the Einstein-Hilbert action, with the help of which we derive the generalized vacuum Einstein equations. When the equations are projected to space-time
\end{abstract}

\footnotetext{
*Corresponding author. E-mail: T.Miller@mini.pw.edu.pl
} 
(giving the "general relativistic limit"), the extra terms that appear due to our generalization can be interpreted as "matter terms", as in Kaluza-Klein-type models. To illustrate this effect we further simplify the metric matrix to a block diagonal form, compute for it the generalized Einstein equations and find two of their "Friedmann-like" solutions for the special case when $G=\mathbb{Z}_{2}$. One of them gives the flat Minkowski space-time (which, however, is not static), another, a hyperbolic, linearly expanding universe.

PACS Nos.: 02.40.Gh, 04.50.-h, 04.20.Jb

\section{Introduction}

In a series of papers ([1, 2, 3] some others will be cited below) we have proposed a model unifying general relativity and quantum mechanics. The core idea of the model consists of an attempt to deduce both general relativity and quantum mechanics from a noncommutative algebra $\mathcal{A}_{\Gamma}$ defined on a transformation groupoid $\Gamma$ determined by the action of the Lorentz group (or one of its subgroups) on the frame bundle $\left(E, \pi_{M}, M\right)$ over space-time $M$. The gravitational sector of the model is given by the noncommutative geometry based on the algebra $\mathcal{A}_{\Gamma}$ and a submodule $V$ of the module of its derivations $\operatorname{Der} \mathcal{A}_{\Gamma}$ (we call the pair $\left(\mathcal{A}_{\Gamma}, V \subseteq \operatorname{Der} \mathcal{A}_{\Gamma}\right)$ differential algebra). The quantum sector of the model is given by the regular representation $\pi: \mathcal{A}_{\Gamma} \rightarrow \mathcal{B}(\mathcal{H})$ of the algebra $\mathcal{A}_{\Gamma}$ on a bundle $\mathcal{H}$ of Hilbert spaces. It turns out that the model has a rich mathematical structure [4], surprising conceptually unifying power [5], and throws some light on fundamental problems of physics [6].

It would certainly be desirable to look at our model in its complete generality by considering the "full" differential algebra $\left(\mathcal{A}_{\Gamma}\right.$, Der $\left.\mathcal{A}_{\Gamma}\right)$. However, this should be preceded by a systematic study of the very rich module of all derivations Der $\mathcal{A}_{\Gamma}$. While this study is in progress, the aim of the present work is much more modest. In the present paper, we investigate a "toy version" of our model, in which the principal bundle is trivial $E=M \times G$ with the base space $M$ compact and the structure group $G$ finite. The transformation groupoid algebra is thus $C^{\infty}(M \times G \times G)$, which in turn is isomorphic to the algebra $\mathbb{M}_{n}\left(C^{\infty}(M)\right)=C^{\infty}(M) \otimes \mathbb{M}_{n}(\mathbb{C})$, where $n$ is the order of the group $G$. In this paper, we limit ourselves to the study of the gravitational sector of this model. Although extremely simple when compared with 
the one studied, for example, in [4, 5], this toy model shows many interesting features; only some of them have been visible in our previous works [7, 8].

Let us add one more motivation of a general nature for studying geometrical properties of the algebras $C^{\infty}(M) \otimes \mathbb{M}_{n}(\mathbb{C})$. The celebrated Gel'fandNaimark theorem states that every commutative and unital $C^{*}$-algebra $\mathcal{C}$ is isometrically isomorphic to the algebra $C(X)$ of complex continuous functions on a compact Hausdorff space $X$. This topological space $X$ can be retrieved from $\mathcal{C}$ by defining it either as the set of all maximal ideals of $\mathcal{C}$ (with the so-called hull-kernel topology) or as the set of characters of $\mathcal{C}$ (with the so-called Gel'fand topology) 1 . As is well known, the very idea of noncommutative geometry relies on this fundamental result [9, 10, 11, 12, 13, 14] . The notion of a "noncommutative space" comes indirectly into being through the study of noncommutative $C^{*}$-algebras that are now interpreted as algebras of "functions" living on this "virtual space". In general, this space can no longer be thought of as consisting of points, since a given noncommutative $C^{*}$-algebra might possess too few maximal ideals as well as too few (or no) characters.

One can thus say that a $C^{*}$-algebra is an adequate algebraic object to encode the notion of a (possibly "noncommutative") set with a certain topological structure. However, in order to study differential geometry and, specifically, general relativity, one needs more, namely a smooth structure and a pseudo-Riemannian structure. The most important example of an algebraical object that is able to encode these structures 2 is a spectral triple, conceived first by Connes [9]. However, the approach we follow does not fall under this scheme, we thus have to look for another method of implementing the smoothness postulate.

First of all, it is reasonable to assume that $\mathcal{A}$ is a dense subalgebra of some $C^{*}$-algebra $\left(\mathcal{C},\|\cdot\|_{\mathcal{C}}\right)$. In other words, $\overline{\mathcal{A}}^{\|\cdot\|_{\mathcal{C}}}=\mathcal{C}$. In this way, in a commutative case, one can readily employ the Gel'fand-Naimark result and, after interpreting $\mathcal{C}$ as a space $C(X)$ of continuous functions on a compact topological space $X$, one can also treat $\mathcal{A}$ as a dense subalgebra of $C(X)$. The idea would be to consider functions from $\mathcal{A}$ as smooth by definition. Of course, not every subalgebra of $C(X)$ is able to encode the smooth structure properly (the subalgebra has to be "rich enough"). One could, for example,

\footnotetext{
${ }^{1}$ These two definitions (or rather ways of constructing $X$ ) are equivalent by Gel'fandMazur theorem.

${ }^{2}$ At least in the Riemannian case.
} 
employ techniques of the theory of differential spaces and demand that this subalgebra be closed with respect to superposition with smooth Euclidean functions [15, 16]. Unfortunately, it is not clear how to extend this condition to the noncommutative setting. We thus propose the following approach.

For a compact Hausdorff space $X$, the vector space $C^{\infty}(X)$ can be equipped with the locally convex topology induced by the family of seminorms $\left\{\|\cdot\|_{D}\right\}$ defined as

$$
\forall f \in C^{\infty}(X) \quad\|f\|_{D}:=\sup _{x \in X}|D f(x)|
$$

where $D$ denotes a differential operator, i.e. an endomorphism of $C^{\infty}(X)$ that can be defined as a linear combination of superpositions of finitely many elements of Der $C^{\infty}(X)$. Note that the identity map $\operatorname{id}_{C^{\infty}(X)}$, regarded as a superposition of zero derivations, is also a differential operator. The set of all differential operators on $C^{\infty}(X)$ can also be obtained as the universal enveloping algebra of the Lie algebra $\operatorname{Der} C^{\infty}(X)$ and as such, will be denoted $U\left(\operatorname{Der} C^{\infty}(X)\right)$.

It is well known that the space $C^{\infty}(X)$ with the topology defined in this way is a complete topological vector space. It is thus natural to formulate the following definition.

We say that a unital differential algebra $(\mathcal{A}, V)$ is a smooth pre-C $C^{*}$-algebra iff $\mathcal{A}$ is a dense subalgebra of a $C^{*}$-algebra $(\mathcal{C},\|\cdot\|)$ complete in the topology induced by the family of seminorms $\left\{\|\cdot\|_{D} \mid D \in U(V)\right\}$ defined through

$$
\forall D \in U(V) \quad \forall a \in \mathcal{A} \quad\|a\|_{D}:=\|D a\| .
$$

Note that this family of seminorms includes the "base" norm, because

$$
\|\cdot\|=\|\cdot\|_{\mathrm{id}_{\mathcal{A}}} \text {. }
$$

Let us further observe that the $C^{*}$-algebra $\mathcal{C}$ can itself be regarded as a smooth pre- $C^{*}$-algebra $(\mathcal{C}, V:=\{0\})$. However, this also means that the smooth structure of $\mathcal{C}$ is trivial (the subject of smooth pre- $C^{*}$-algebras will be addressed in a forthcoming paper).

Let us finally notice that the algebra $\mathcal{A}_{n}:=C^{\infty}(M) \otimes \mathbb{M}_{n}(\mathbb{C})$ with the full module of its derivations is a smooth pre- $C^{*}$-algebra. Therefore, by investigating its geometrical properties we in fact study a basic realization of a smooth pre- $C^{*}$-algebra-based noncommutative geometry. 
The algebras $\mathcal{A}_{n}$ have yet another interesting property. Their maximal ideals are of the form $J_{m} \otimes \mathbb{M}_{n}(\mathbb{C})$, where $m \in M$, and $J_{m}$ is an ideal of $C^{\infty}(M)$ consisting of all smooth functions that vanish at $m$. Therefore, there is a bijection between $M$ and the set of maximal ideals of the noncommutative algebra $\mathcal{A}_{n}$. One can thus say, that although the noncommutative space associated with the algebra $\mathcal{A}_{n}$ does not consist of points, it nevertheless allows one to retrieve the point space $M$ associated with the center of the algebra $\mathcal{A}_{n}$, its "commutative ingredient" $C^{\infty}(M) \otimes\left\{I_{n}\right\} \simeq C^{\infty}(M)$ where $I_{n}$ denotes an " $n$ by $n$ " identity matrix.

The plan of our paper runs as follows. In Section 2, we present the basic structure of the model, focusing on its elements that are new as compared with previous versions of the model. We analyse, in some detail, the module Der $\mathcal{A}_{n}$ of derivations of the groupoid algebra $\mathcal{A}_{n}$ as preparation for the study, in Section 3, of the geometry of the differential algebra, understood as the pair $\left(\mathcal{A}_{n}\right.$, Der $\left.\mathcal{A}_{n}\right)$. In Section 4 , we define the Einstein-Hilbert action for our model, with the help of which we derive the generalized vacuum Einstein equations. Interestingly, when the equations are projected to space-time (giving the "general relativistic limit"), the extra terms that appear due to our generalization, can be interpreted as "matter terms", not unlike in KaluzaKlein-type models [17, 18, 19, 20]. We illustrate this effect in Section 5, by constructing the simplified model with the metric that does not "mix" horizontal and inner derivations (the metric matrix has a block diagonal form). For this case, we find two "Friedmann-like" solutions for the special case when $G=\mathbb{Z}_{2}$. Finally, in Section 6 , we collect some comments and interpretative remarks.

\section{Groupoid algebra and its derivations.}

In the following, we shall consider a simplified version of the groupoid unification model, in which the Lorentz group is replaced with a finite group $G=\left\{e=g_{1}, g_{2}, g_{3}, \ldots, g_{n}\right\}$. This implies that the smooth principal bundle $E$ is trivial, $E=M \times G$. We will also assume that the base space $M$ of the bundle is a compact manifold of dimension $N$. Therefore, the corresponding transformation groupoid is $\Gamma=E \times G=M \times G \times G$, and the convolution 
in the space $C^{\infty}(\Gamma)$ of smooth complex functions on $\Gamma$ is given by

$$
\forall a, b \in C^{\infty}(\Gamma) \quad(a * b)\left(x, g_{k}, g_{l}\right):=\sum_{m=1}^{n} a\left(x, g_{k}, g_{m}\right) b\left(x, g_{k} g_{m}, g_{m}^{-1} g_{l}\right) \text {. }
$$

However, by introducing the following notation

$$
\forall a \in C^{\infty}(\Gamma) \quad a_{i j}(x):=a\left(x, g_{i}, g_{i}^{-1} g_{j}\right)
$$

one can easily recover from (11) the formula for matrix multiplication 3

$$
\forall a, b \in C^{\infty}(\Gamma) \quad(a * b)_{i j}(x):=\sum_{k=1}^{n} a_{i k}(x) b_{k j}(x) .
$$

The convolution algebra $\left(C^{\infty}(\Gamma), *\right)$ can thus be identified with the algebra $\mathcal{A}_{n}$ of " $n$ by $n$ " matrices with entries from the algebra $C^{\infty}(M)$ of smooth functions on the manifold $M$ (with pointwise multiplication)

$$
\mathcal{A}_{n}:=\mathbb{M}_{n}\left(C^{\infty}(M)\right)=C^{\infty}(M) \otimes \mathbb{M}_{n}(\mathbb{C}) .
$$

The center $Z\left(\mathcal{A}_{n}\right)$ of this algebra consists of matrices of the form $f I_{n}$, where $f \in C^{\infty}(M)$. It is therefore isomorphic to the algebra $C^{\infty}(M)$.

As we briefly explained in the introduction, our approach to noncommutative geometry of the algebra $\mathcal{A}_{n}$ is based on the $Z\left(\mathcal{A}_{n}\right)$-module of its derivations, denoted Der $\mathcal{A}_{n}$. Let us recall that a derivation of a $\mathbb{C}$-algebra $\left(\mathcal{A}_{n}, *\right)$ is a $\mathbb{C}$-linear map $d: \mathcal{A}_{n} \rightarrow \mathcal{A}_{n}$ satisfying the Leibniz rule, namely,

$$
\forall a, b \in \mathcal{A}_{n} \quad d(a * b)=d a * b+a * d b .
$$

It is straightforward to realize that the set Der $\mathcal{A}_{n}$ is indeed a $Z\left(\mathcal{A}_{n}\right)$ module. It also possesses the structure of a Lie $\mathbb{C}$-algebra with the Lie bracket given by the commutator

$$
\forall d_{1}, d_{2} \in \operatorname{Der} \mathcal{A}_{n} \quad\left[d_{1}, d_{2}\right]:=d_{1} \circ d_{2}-d_{2} \circ d_{1} .
$$

It is well known 4 that in the case of our matrix algebra $\mathcal{A}_{n}$, the module of its derivations can be written as a direct sum of the submodules of horizontal and inner derivations

$$
\operatorname{Der} \mathcal{A}_{n}=\operatorname{Hor} \mathcal{A}_{n} \oplus \operatorname{Inn} \mathcal{A}_{n}
$$

\footnotetext{
${ }^{3}$ Another way of realizing this fact is by considering a suitable pair groupoid [4, 5, 7].

${ }^{4}$ In fact, one can treat this result as a corollary of a much more general theorem concerning derivations of tensor products of algebras [21].
} 
Horizontal derivations are defined as liftings of the smooth vector fields on the manifold $M$ onto $\mathcal{A}_{n}$. More explicitly, for any smooth vector field $X \in \operatorname{Der} C^{\infty}(M)$ one can define its lifting $\bar{X} \in \operatorname{Der} \mathcal{A}_{n}$ as a map acting entrywise

$$
\forall a \in \mathcal{A}_{n} \quad \bar{X} a:=\left(X a_{i j}\right)_{i j} .
$$

In fact, the lifting map ${ }^{-}: \operatorname{Der} C^{\infty}(M) \rightarrow$ Hor $\mathcal{A}_{n}$ is an isomorphism both in the sense of $C^{\infty}(M)$-modules (where from now on we shall identify the center $Z\left(\mathcal{A}_{n}\right)$ with $\left.C^{\infty}(M)\right)$ and in the sense of Lie $\mathbb{C}$-algebras 5 .

This means that Hor $\mathcal{A}_{n}$ is a locally free finitely generated $C^{\infty}(M)$-module, by which we mean that for any fixed chart $(U, \chi)$ on the manifold $M$, every element $\bar{X} \in \operatorname{Hor} \mathcal{A}_{n}$ can be restricted (or "localized") to a derivation $\left.\bar{X}\right|_{U} \in \operatorname{Der} C^{\infty}(U \times G \times G)$, which in turn can be expressed as a $C^{\infty}(U)$ linear combination of the liftings $\frac{\partial}{\partial \chi^{\mu}}$ of the local vector fields induced by the chart $\chi$. Symbolically,

$$
\left.\left(\operatorname{Hor} \mathcal{A}_{n}\right)\right|_{U}=\operatorname{span}_{C^{\infty}(U)}\left(\overline{\frac{\partial}{\partial \chi^{\mu}}}\right)_{\mu=0,1, \ldots, N-1}
$$

In the following, we shall denote the liftings of the local vector fields $\overline{\frac{\partial}{\partial \chi^{\mu}}}$ simply $\partial_{\mu}$, suppressing both the overline and the reference to the inducing chart.

By an inner derivation induced by an element $b \in \mathcal{A}_{n}$ one understands a $\operatorname{map} \operatorname{ad}_{b}: \mathcal{A}_{n} \rightarrow \mathcal{A}_{n}$ defined as

$$
\forall a \in \mathcal{A}_{n} \quad \operatorname{ad}_{b} a:=[b, a]=b * a-a * b .
$$

The $C^{\infty}(M)$-submodule $\operatorname{Inn} \mathcal{A}_{n}$ of all inner derivations of $\mathcal{A}_{n}$ possesses also the structure of a Lie algebra which, moreover, is an ideal of the Lie algebra Der $\mathcal{A}_{n}$.

It is straightforward to show that the map ad $: \mathcal{A}_{n} \rightarrow \operatorname{Inn} \mathcal{A}_{n}, b \mapsto \operatorname{ad}_{b}$, is an epimorphism in the sense of $C^{\infty}(M)$-modules, as well as an epimorphism going from the Lie algebra associated 6 to $\mathcal{A}_{n}$ onto the Lie algebra $\operatorname{Inn} \mathcal{A}_{n}$, its

\footnotetext{
${ }^{5}$ Note that Hor $\mathcal{A}_{n}$ possesses the structure of a commutative Lie algebra.

${ }^{6}$ For a given algebra $(\mathcal{A}, *)$, its associated Lie algebra $(\mathcal{A},[.,]$.$) is the underlying vector$ space of $\mathcal{A}$ equipped with a Lie bracket given by the commutator $[a, b]:=a * b-b * a$ for any $a, b \in \mathcal{A}$.
} 
kernel being the center of $\mathcal{A}_{n}$. By using the First Isomorphism Theorem, one easily obtains an isomorphism between the quotient $\mathcal{A}_{n} / Z\left(\mathcal{A}_{n}\right)$ (possessing both the structure of a $C^{\infty}(M)$-module and of a Lie algebra) and $\operatorname{Inn} \mathcal{A}_{n}$. This quotient space, in turn, is itself isomorphic to the space $\mathfrak{s l}_{n}\left(C^{\infty}(M)\right)$ of traceless " $n$ by $n$ " matrices with entries from $C^{\infty}(M)$, again both in the sense of $C^{\infty}(M)$-modules and in the sense of Lie algebras. All in all, we can write

$$
\begin{aligned}
\operatorname{Inn} \mathcal{A}_{n} & =\operatorname{ad}\left(\mathcal{A}_{n}\right) \cong \mathcal{A}_{n} / Z\left(\mathcal{A}_{n}\right) \\
& =\mathbb{M}_{n}\left(C^{\infty}(M)\right) /\left\{f I_{n} \mid f \in C^{\infty}(M)\right\} \cong \mathfrak{s l}_{n}\left(C^{\infty}(M)\right) .
\end{aligned}
$$

With a slight abuse of notation, we shall denote the isomorphism between $\mathfrak{s l}_{n}\left(C^{\infty}(M)\right)$ and $\operatorname{Inn} \mathcal{A}_{n}$ also by ad. With this isomorphism at hand, it is clear that $\operatorname{Inn} \mathcal{A}_{n}$ is a free finitely generated $C^{\infty}(M)$-module, whose dimension is equal to $n^{2}-1$. Its basis might be determined by the choice of the basis in $\mathfrak{s l}_{n}\left(C^{\infty}(M)\right)$. We choose the latter in the following way.

Let $E_{i j}$ denote the " $n$ by $n$ " matrix of the form $E_{i j}:=\left(\delta_{i k} \delta_{j l}\right)_{k, l}$. Taking only those matrices $E_{i j}$ for which $k \neq l$ together with the matrices $D_{k}:=E_{k k}-E_{k+1, k+1},(k=1,2, \ldots, n-1)$, one obtains a convenient basis in the $C^{\infty}(M)$-module $\mathfrak{s l}_{n}\left(C^{\infty}(M)\right)$.

Now, let us denote $\partial_{\{i j\}}:=\operatorname{ad}_{E_{i j}}$, for $i, j=1,2, \ldots, n$, where $i \neq j$ and $\partial_{\{k\}}:=\operatorname{ad}_{D_{k}}$ for $k=1,2, \ldots, n-1$. These derivations form a basis of the $C^{\infty}(M)$-module Inn $\mathcal{A}_{n}$. Symbolically,

$$
\operatorname{Inn} \mathcal{A}_{n}=\operatorname{span}_{C^{\infty}(M)}\left(\left(\partial_{\{i j\}}\right)_{i, j=1, \ldots, n ; i \neq j} \cup\left(\partial_{\{k\}}\right)_{k=1, \ldots, n-1}\right) .
$$

Summarizing, Der $\mathcal{A}_{n}$ is thus a locally free finitely generated $C^{\infty}(M)$ module; its local basis consists of the derivations of three kinds, namely horizontal derivations and two sorts of inner derivations. To denote them we use the same symbol " $\partial$ " indexed according to the following key

- Horizontal derivations are indexed by the lowercase Greek letters $\mu, \nu, \lambda, \ldots$ assuming the values $0,1, \ldots, N-1$ ( $N$ stands for the dimension of the manifold $M$ ).

- Inner derivations induced by the matrices $E_{i j}$ are indexed by the pairs of lowercase Latin letters in curly brackets $\{i j\},\{k l\},\{p q\}, \ldots$ assuming the values $\{12\},\{13\}, \ldots,\{1 n\},\{21\},\{23\}, \ldots,\{n, n-1\}$ (that is, out of all possible pairs only the pairs $\{11\},\{22\}, \ldots,\{n n\}$ are excluded). 
- Inner derivations induced by the matrices $D_{k}$ are indexed by the single lowercase Latin letters in curly brackets $\{a\},\{b\},\{c\}, \ldots$ assuming the values $\{1\},\{2\}, \ldots,\{n-1\}$.

- Additionally, it is convenient to use the capital Latin letters $A, B, C, \ldots$ to index all derivations in the basis; these indices assume all the values listed earlier.

- Finally, the capital Latin letters in curly brackets $\{A\},\{B\},\{C\}, \ldots$ index all inner derivations, taking all values assumed by the indices of the types $\{i j\}$ and $\{a\}$

Let us illustrate the above introduced notation to describe the Lie algebra structure of Der $\mathcal{A}_{n}$.

Claim. For the elements of the local basis of Der $\mathcal{A}_{n}$, the following commutation rules hold

$\left[\partial_{\mu}, \partial_{\nu}\right]=0, \quad\left[\partial_{\mu}, \partial_{\{b\}}\right]=0, \quad\left[\partial_{\mu}, \partial_{\{k l\}}\right]=0, \quad\left[\partial_{\{a\}}, \partial_{\{b\}}\right]=0$, $\left[\partial_{\{a\}}, \partial_{\{k l\}}\right]=\delta_{k a} \partial_{\{a l\}}-\delta_{a l} \partial_{\{k a\}}+\delta_{a+1, l} \partial_{\{k, a+1\}}-\delta_{k, a+1} \partial_{\{a+1, l\}}$,

$\left[\partial_{\{i j\}}, \partial_{\{k l\}}\right]= \begin{cases}\partial_{\{i l\}} & \text { for } i \neq l \wedge k=j, \\ -\partial_{\{k j\}} & \text { for } i=l \wedge k \neq j, \\ \partial_{\{i\}}+\partial_{\{i+1\}}+\ldots+\partial_{\{j-1\}} & \text { for } i=l \wedge k=j \wedge i<j, \\ -\left(\partial_{\{j\}}+\partial_{\{j+1\}}+\ldots+\partial_{\{i-1\}}\right) & \text { for } i=l \wedge k=j \wedge i>j, \\ 0 & \text { for } i \neq l \wedge k \neq j .\end{cases}$

The claim is a straightforward consequence of the following commutation rule for the matrices $E_{i j}$ :

$$
E_{i j} E_{k l}-E_{k l} E_{i j}=\delta_{k j} E_{i l}-\delta_{i l} E_{k j}
$$

In other words, because of the noncommutativity of $\mathcal{A}_{n}$, some of the struc- 
ture constants $\mathbf{c}_{A B}{ }^{C}$ of the Lie algebra Der $\mathcal{A}_{n}$ are nontrivial. To be precise,

$$
\begin{aligned}
& \mathbf{c}_{\{a\}\{k l\}}^{\{p q\}}=\delta_{p a} \delta_{q l} \delta_{k a}-\delta_{p k} \delta_{q a} \delta_{a l}+\delta_{p k} \delta_{q, a+1} \delta_{a+1, l}-\delta_{p, a+1} \delta_{q, l} \delta_{k, a+1}, \\
& \mathbf{c}_{\{i j\}\{b\}}{ }_{\{p q\}}=-\mathbf{c}_{\{b\}\{i j\}}{ }_{\{p q\}}, \\
& \mathbf{c}_{\{i j\}\{k l\}}=\delta_{p i} \delta_{q l} \delta_{k j}-\delta_{p k} \delta_{q j} \delta_{i l}, \\
& \mathbf{c}_{\{i j\}\{k l\}}^{\{c\}}= \begin{cases}\delta_{i l} \delta_{k j}\left(\delta_{c i}+\delta_{c, i+1}+\ldots+\delta_{c, j-1}\right) & \text { for } i<j, \\
-\delta_{i l} \delta_{k j}\left(\delta_{c j}+\delta_{c, j+1}+\ldots+\delta_{c, i-1}\right) & \text { for } i>j,\end{cases}
\end{aligned}
$$

and all other structure constants are zero.

To further get used to the notation, let us consider an example in which $G=\mathbb{Z}_{2}$. The algebra we study is then $\mathcal{A}_{2}:=\mathbb{M}_{2}\left(C^{\infty}(M)\right)=C^{\infty}(M) \otimes$ $\mathbb{M}_{2}(\mathbb{C})$. In this case, the $C^{\infty}(M)$-module $\operatorname{Inn} \mathcal{A}_{2}$ is spanned by three derivations: $\partial_{\{12\}}, \partial_{\{21\}}$ and $\partial_{\{1\}}$, that are induced by the following matrices, respectively:

$$
E_{12}=\left[\begin{array}{ll}
0 & 1 \\
0 & 0
\end{array}\right], \quad E_{21}=\left[\begin{array}{ll}
0 & 0 \\
1 & 0
\end{array}\right], \quad D_{1}=\left[\begin{array}{cc}
1 & 0 \\
0 & -1
\end{array}\right] .
$$

One has the following commutation relations:

$$
\left[\partial_{\{1\}}, \partial_{\{12\}}\right]=2 \partial_{\{12\}}, \quad\left[\partial_{\{1\}}, \partial_{\{21\}}\right]=-2 \partial_{\{21\}}, \quad\left[\partial_{\{12\}}, \partial_{\{21\}}\right]=\partial_{\{1\}} .
$$

These relations translate into the following values of the nonzero structure constants:

$$
\begin{aligned}
\left.\mathbf{c}_{\{1\}\{12\}}\right\}^{\{12\}} & \left.=-\mathbf{c}_{\{12\}\{1\}}\right\}^{212\}}=2, \\
\mathbf{c}_{\{1\}\{21\}}{ }^{\{21\}}=-\mathbf{c}_{\{21\}\{1\}}\{21\} & =-2, \\
\mathbf{c}_{\{12\}\{21\}}{ }^{\{1\}}=-\mathbf{c}_{\{21\}\{12\}}\{1\} & =1 .
\end{aligned}
$$

\section{Geometry of the differential algebra $\left(\mathcal{A}_{n}\right.$, Der $\left.\mathcal{A}_{n}\right)$.}

In this section, we construct the elements of the pseudo-Riemannian geometry of the algebra $\mathcal{A}_{n}$. We proceed along the lines first proposed by Parfionov

\footnotetext{
${ }^{7}$ The structure constants $\mathbf{c}_{A B}{ }^{C}$, here interpreted as constant functions on $M$, are defined by $\left[\partial_{A}, \partial_{B}\right]=\mathbf{c}_{A B}{ }^{C} \partial_{C}$.
} 
and Zapatrin [22] and further developed in [4, 16, 23]. Our "toy version" of the model, albeit simple, still offers a nontrivial extension of the standard pseudo-Riemannian geometry.

For brevity, let us denote the $C^{\infty}(M)$-module $\operatorname{Der}\left(\mathcal{A}_{n}\right)$ by $V$. We define $V^{*} \equiv \operatorname{Hom}_{C^{\infty}(M)}\left(V, C^{\infty}(M)\right)$ to be its dual.

Let now $\mathcal{G}: V \times V \rightarrow C^{\infty}(M)$ be a symmetric $C^{\infty}(M)$-bilinear map, the metric in our model. We assume that $\mathcal{G}$ is nondegenerate, that is, that the map $\Phi_{\mathcal{G}}: V \rightarrow V^{*}$, given by

$$
\forall u, v \in V \quad \Phi_{\mathcal{G}}(u)(v)=\mathcal{G}(u, v),
$$

is an isomorphism of $C^{\infty}(M)$-modules.

For the sake of further calculations, it is convenient to express the metric in the local basis of $V$ introduced in the previous section. We thus have the metric matrix $\left(g_{A B}\right)$ with the entries

$$
g_{A B}:=\mathcal{G}\left(\partial_{A}, \partial_{B}\right) .
$$

It is a square, symmetric and nonsingular matrix of order $N+n^{2}-1$. We denote its inverse matrix by $\left(g^{A B}\right)$. Exactly as in the standard case, the metric matrix and its inverse can be used to lower or raise indices. However, one should remember that this concerns only the indices of the type $A, B, C, \ldots 8$.

For instance, we shall be using the completely covariant structure constants $\mathbf{c}_{A B C}:=\mathbf{c}_{A B}{ }^{D} g_{D C}$. For our algebra, they can be expressed as

$$
\begin{aligned}
& \mathbf{c}_{\{a\}\{k l\} C}=\delta_{k a} g_{\{a l\} C}-\delta_{a l} g_{\{k a\} C}+\delta_{a+1, l} g_{\{k, a+1\} C}-\delta_{k, a+1} g_{\{a+1, l\} C}, \\
& \mathbf{c}_{\{i j\}\{b\} C=}=-\mathbf{c}_{\{b\}\{i j\} C}, \\
& \mathbf{c}_{\{i j\}\{k l\} C}= \begin{cases}\delta_{i l} \delta_{k j}\left(g_{\{i\} C}+g_{\{i+1\} C}+\ldots+g_{\{j-1\} C}\right) \\
+\delta_{k j} g_{\{i l\} C}-\delta_{i l} g_{\{k j\} C} \\
-\delta_{i l} \delta_{k j}\left(g_{\{j\} C}+g_{\{i+1\} C}+\ldots+g_{\{i-1\} C}\right) \\
+\delta_{k j} g_{\{i l\} C}-\delta_{i l} g_{\{k j\} C} & \text { for } i<j,\end{cases}
\end{aligned}
$$

with all the remaining ones equal to zero.

We are now ready to define the Levi-Civita preconnection $\nabla^{*}: V \times V \rightarrow$ $V^{*}$ by using the Koszul formula

$$
\begin{aligned}
\left(\nabla_{u}^{*} v\right)(w): & =\frac{1}{2}[u(\mathcal{G}(v, w))+v(\mathcal{G}(u, w))-w(\mathcal{G}(u, v)) \\
& +\mathcal{G}(w,[u, v])+\mathcal{G}(v,[w, u])-\mathcal{G}(u,[v, w])]
\end{aligned}
$$

\footnotetext{
${ }^{8}$ Unless the metric matrix is block diagonal, see Section 5.
} 
for any $u, v, w \in V$.

The components $\Gamma_{A B C}$ of the Levi-Civita preconnection in the local basis (the Christoffel symbols of the first kind) are expressed by the following general formula:

$$
\begin{aligned}
\Gamma_{A B C} & :=\left(\nabla_{\partial_{C}}^{*} \partial_{B}\right)\left(\partial_{A}\right) \\
& =\frac{1}{2}\left(\partial_{C} g_{A B}+\partial_{B} g_{A C}-\partial_{A} g_{B C}+\mathbf{c}_{C B A}+\mathbf{c}_{A C B}-\mathbf{c}_{B A C}\right)
\end{aligned}
$$

Let us notice that, unlike in the standard case, the components $\Gamma_{A B C}$ are in general not symmetric with respect to interchanging the second and third indices,

$$
\Gamma_{A B C}-\Gamma_{A C B}=\mathbf{c}_{C B A}
$$

The Levi-Civita connection $\nabla: V \times V \rightarrow V$ is defined as

$$
\nabla:=\Phi_{\mathcal{G}}^{-1} \circ \nabla^{*}
$$

Its components in the local basis (the Christoffel symbols of the second kind) are exactly the components of the Levi-Civita preconnection with the first index raised $\Gamma_{B C}^{A}$. This means that they can be equivalently defined by the equality

$$
\nabla_{\partial_{C}} \partial_{B}=\Gamma_{B C}^{A} \partial_{A}
$$

and that they can be expressed as

$$
\Gamma_{B C}^{A}=\frac{1}{2} g^{A D}\left(\partial_{C} g_{D B}+\partial_{B} g_{D C}-\partial_{D} g_{B C}+\mathbf{c}_{C B D}+\mathbf{c}_{D C B}-\mathbf{c}_{B D C}\right) .
$$

Just as for the preconnection components, we have the asymmetry

$$
\Gamma_{B C}^{A}-\Gamma_{C B}^{A}=\mathbf{c}_{C B}^{A} .
$$

The Levi-Civita connection enjoys all the properties of its standard coun- 
terpart, that is 9

$$
\begin{array}{ll}
1^{\circ} & \nabla_{u+v} w=\nabla_{u} w+\nabla_{v} w \\
2^{\circ} & \nabla_{f u} v=f \nabla_{u} v \\
3^{\circ} & \nabla_{u}(v+w)=\nabla_{u} v+\nabla_{u} w \\
4^{\circ} & \nabla_{u}(f v)=u(f) v+f \nabla_{u} v, \\
5^{\circ} & \nabla_{u} v-\nabla_{v} u-[u, v]=0 \\
& (\text { torsion-freeness }) \\
6^{\circ} & w(\mathcal{G}(u, v))=\mathcal{G}\left(\nabla_{w} u, v\right)+\mathcal{G}\left(u, \nabla_{w} v\right) \\
& (\text { metric compatibility })
\end{array}
$$

for all $u, v, w \in V$ and $f \in C^{\infty}(M)$. Moreover, just like in the standard case, the Levi-Civita connection is the unique map $V \times V \rightarrow V$ that satisfies $5^{\circ}$ and $6^{\circ}$.

With the help of the Levi-Civita connection, one can introduce the curvature tensors. The Riemann curvature tensor $R: V \times V \times V \rightarrow V$, $(u, v, w) \mapsto R(u, v) w$ is defined as

$$
R(u, v) w:=\nabla_{u} \nabla_{v} w-\nabla_{v} \nabla_{u} w-\nabla_{[u, v]} w .
$$

Its components $R_{D A B}^{C}$ in the local basis of $V$, defined by the formula

$$
R\left(\partial_{A}, \partial_{B}\right) \partial_{D}=R_{D A B}^{C} \partial_{C}
$$

can be expressed as

$$
R_{D A B}^{C}=\partial_{A} \Gamma_{D B}^{C}-\partial_{B} \Gamma_{D A}^{C}+\Gamma_{D B}^{K} \Gamma_{K A}^{C}-\Gamma_{D A}^{K} \Gamma_{K B}^{C}-\mathbf{c}_{A B}{ }^{K} \Gamma_{D K}^{C} .
$$

The map $R$ enjoys the usual Riemann tensor symmetries which, when expressed in the local basis, read

$$
\begin{aligned}
& R_{C D A B}=-R_{D C A B}=-R_{C D B A}=R_{A B C D}, \\
& R_{D A B}^{C}+R_{B D A}^{C}+R_{A B D}^{C}=0 .
\end{aligned}
$$

Thanks to the finite-dimensionality of $V$, one can use the standard tensor contraction operation to define the Ricci tensor, ric : $V \times V \rightarrow C^{\infty}(M)$, and

\footnotetext{
${ }^{9}$ Properties $1^{\circ}-6^{\circ}$ follow directly from the Koszul formula (9).
} 
the curvature scalar $r \in C^{\infty}(M)$. The components of the Ricci tensor in the local basis read

$$
\operatorname{ric}_{A B}:=R_{A C B}^{C}
$$

and the curvature scalar is

$$
r:=g^{A B} \mathbf{r i c}_{A B}=g^{A B} R_{A C B}^{C}
$$

Note that ric is a symmetric tensor, $\mathbf{r i c}_{A B}=\mathbf{r i c}_{B A}$.

Having defined all the basic pseudo-Riemannian-geometric elements of the "toy version" of our model, we are ready to investigate some of its relativistic aspects.

\section{Generalized Einstein equations from the ac- tion principle.}

In this Section, after providing a straightforward generalization of the EinsteinHilbert action, we derive the generalized vacuum Einstein equations.

Let us consider the following Einstein-Hilbert action functional

$$
S_{E H}:=\int r \sqrt{|g|} d^{N} x
$$

where again $N=\operatorname{dim} M$ and $g$ denotes the determinant of the metric matrix $\left(g_{A B}\right)$. We postulate no additional matter term.

Let us vary $S_{E H}$ with respect to $\delta g^{A B}$

$$
\begin{aligned}
\delta S_{E H}= & \int\left(\operatorname{ric}_{A B}-\frac{1}{2} r g_{A B}\right) \delta g^{A B} \sqrt{|g|} d^{N} x \\
& +\int \delta \operatorname{ric}_{A B} g^{A B} \sqrt{|g|} d^{N} x .
\end{aligned}
$$

We will now show that the rightmost integral in (19) can be omitted, because its integrand can be expressed as a divergence, namely

$$
\delta \text { ric }_{A B} g^{A B} \sqrt{|g|}=\partial_{\mu}\left[\left(g^{A B} \delta \Gamma_{A B}^{\mu}-g^{\mu B} \delta \Gamma_{B A}^{A}\right) \sqrt{|g|}\right] .
$$

We begin the proof of this statement by making the following observations that will greatly simplify further computations. 
(i) The structure constants $\mathbf{c}_{A B}{ }^{C}$ do not depend on the components of the metric matrix $g_{A B}$, and hence their variations vanish

$$
\delta \mathbf{c}_{A B}{ }^{C}=0 .
$$

(ii) The structure constants $\mathbf{c}_{A B}{ }^{C}$ do not depend on the space-time coordinates, and hence they vanish under the action of any derivation

$$
\partial_{D} \mathbf{c}_{A B}{ }^{C}=0 .
$$

(iii) Any contraction of $\mathbf{c}_{A B}{ }^{C}$ yields zero

$$
\mathbf{c}_{A B}{ }^{A}=0, \quad \mathbf{c}_{A B}{ }^{B}=0, \quad g^{A B} \mathbf{c}_{A B}{ }^{C}=0 .
$$

(iv) Although the Christoffel symbols are in general asymmetric with respect to interchanging the second and third indices (13)

$$
\Gamma_{B C}^{A}-\Gamma_{C B}^{A}=\mathbf{c}_{C B}{ }^{A},
$$

the symmetry is recovered for contractions, derivatives and variations of the symbols

$$
\Gamma_{A C}^{A}=\Gamma_{C A}^{A}, \quad \partial_{D} \Gamma_{B C}^{A}=\partial_{D} \Gamma_{C B}^{A}, \quad \delta \Gamma_{B C}^{A}=\delta \Gamma_{C B}^{A} .
$$

Moreover,

$$
\Gamma_{A C}^{A}=\frac{1}{2} g^{A B} \partial_{C} g_{A B}=-\frac{1}{2} g_{A B} \partial_{C} g^{A B}
$$

To prove (iii) let us directly compute the contraction $\mathbf{c}_{A B}{ }^{B}$, and let us do this separately for all possible kinds of the index $A$. By (2) we obtain

$$
\begin{aligned}
\mathbf{c}_{\mu B}{ }^{B} & =0 \quad \text { (trivially), } \\
\mathbf{c}_{\{a\} B}{ }^{B} & =\mathbf{c}_{\{a\}\{i j\}}\{i j\} \\
& =\sum_{i, j=1}^{n}\left(\delta_{i a} \delta_{j j} \delta_{i a}-\delta_{i i} \delta_{j a} \delta_{a j}+\delta_{i i} \delta_{j, a+1} \delta_{a+1, j}-\delta_{i, a+1} \delta_{j j} \delta_{i, a+1}\right) \\
& =n-n+n-n=0, \\
\mathbf{c}_{\{k l\} B}{ }^{B} & =\mathbf{c}_{\{k l\}\{i j\}}\{i j\} \\
&
\end{aligned}
$$


The other two contractions vanish on the strength of the skew-symmetry $\mathbf{c}_{A B}{ }^{C}=-\mathbf{c}_{B A}{ }^{C}$.

As for (iv), it is a straightforward consequence of (i-iii) and (13).

We are now ready to prove (20). By expanding the expression $\delta \mathbf{r i c}_{A B} g^{A B}$, we obtain

$$
\begin{aligned}
\delta \operatorname{ric}_{A B} g^{A B}= & \delta R_{A C B}^{C} g^{A B} \\
= & g^{A B}\left(\partial_{C} \delta \Gamma_{A B}^{C}-\partial_{B} \delta \Gamma_{A C}^{C}+\delta \Gamma_{A B}^{K} \Gamma_{K C}^{C}+\Gamma_{A B}^{K} \delta \Gamma_{K C}^{C}{ }_{K C}\right. \\
& \left.\quad-\delta \Gamma_{A C}^{K} \Gamma_{K B}^{C}-\Gamma_{A C}^{K} \delta \Gamma_{K B}^{C}-\mathbf{c}_{C B}{ }_{K} \delta \Gamma_{A K}^{C}{ }_{A K}\right)
\end{aligned}
$$

where we have used (i) and the fact that the derivatives of variations are equal to the variations of derivatives.

The last three terms in (21) can be merged into a single term with the help of (13) and (iv). Concretely, one can show that

$$
g^{A B}\left(\delta \Gamma_{A C}^{K} \Gamma_{K B}^{C}+\Gamma_{A C}^{K} \delta \Gamma_{K B}^{C}+\mathbf{c}_{C B}{ }^{K} \delta \Gamma_{A K}^{C}\right)=2 g^{A B} \Gamma_{B C}^{K} \delta \Gamma_{A K}^{C} .
$$

This term, in turn, can be expressed as

$$
2 g^{A B} \Gamma_{B C}^{K} \delta \Gamma_{A K}^{C}=-\partial_{C} g^{K A} \delta \Gamma_{A K}^{C} .
$$

Indeed, by expanding the Christoffel symbol $\Gamma_{B C}^{K}$ one gets

$$
\begin{aligned}
& 2 g^{A B} \Gamma_{B C}^{K} \delta \Gamma_{A K}^{C} \\
& =g^{A B} g^{K D}\left(\partial_{C} g_{D B}+\partial_{B} g_{D C}-\partial_{D} g_{B C}+\mathbf{c}_{C B D}+\mathbf{c}_{D C B}-\mathbf{c}_{B D C}\right) \delta \Gamma_{A K}^{C} \\
& =g^{A B} g^{K D} \partial_{C} g_{D B} \delta \Gamma_{A K}^{C} \\
& \quad+g^{A B} g^{K D}(\underbrace{\partial_{B} g_{D C}-\partial_{D} g_{B C}}_{=0}+\underbrace{\mathbf{c}_{C B D}-\mathbf{c}_{C D B}}_{=0}-\underbrace{\mathbf{c}_{B D C}}_{=0}) \delta \Gamma_{A K}^{C} \\
& =-\partial_{C} g^{K A} \delta \Gamma_{A K}^{C},
\end{aligned}
$$

where all the underbraced terms vanish because the term $g^{A B} g^{K D} \delta \Gamma_{A K}^{C}$ is symmetric with respect to interchanging the indices $B$ and $D$. Moreover, in the last equality we have employed the well-known formula for the derivative of the matrix inverse.

Thus, equality (21) can be rewritten in the form

$$
\begin{aligned}
\delta \operatorname{ric}_{A B} g^{A B}= & g^{A B} \partial_{C} \delta \Gamma_{A B}^{C}-g^{A B} \partial_{B} \delta \Gamma_{A C}^{C}+g^{A B} \delta \Gamma_{A B}^{K} \Gamma_{K C}^{C} \\
& +g^{A B} \Gamma_{A B}^{K}{ }_{A B} \Gamma_{K C}^{C}+\partial_{C} g^{K A} \delta \Gamma_{A K}^{C} .
\end{aligned}
$$


Let us now expand the expression $\frac{1}{\sqrt{|g|}} \partial_{C}\left[\left(g^{A B} \delta \Gamma_{A B}^{C}-g^{C B} \delta \Gamma_{B A}^{A}\right) \sqrt{|g|}\right]$. By using the fact that, by (iv),

$$
\partial_{C} \sqrt{|g|}=\frac{1}{2} \sqrt{|g|} g^{D K} \partial_{C} g_{D K}=\sqrt{|g|} \Gamma_{K C}^{K},
$$

one has

$$
\begin{aligned}
& \frac{1}{\sqrt{|g|}} \partial_{C}\left[\left(g^{A B} \delta \Gamma_{A B}^{C}-g^{C B} \delta \Gamma_{B A}^{A}\right) \sqrt{|g|}\right] \\
& =\partial_{C} g^{A B} \delta \Gamma_{A B}^{C}+g^{A B} \partial_{C} \delta \Gamma_{A B}^{C}-\partial_{C} g^{C B} \delta \Gamma_{B A}^{A}-g^{C B} \partial_{C} \delta \Gamma_{B A}^{A} \\
& \quad+\Gamma_{K C}^{K} g^{A B} \delta \Gamma_{A B}^{C}-\Gamma_{K C}^{K} g^{C B} \delta \Gamma_{B A}^{A} .
\end{aligned}
$$

Comparing (23) with (22) (and renaming some of the dummy indices), one concludes that they are in fact equal if and only if

$$
\left(\Gamma_{K C}^{K} g^{C B}+\Gamma_{C K}^{B} g^{C K}+\partial_{K} g^{K B}\right) \delta \Gamma_{A B}^{A}=0 .
$$

But this is indeed the case because, by direct calculation one gets

$$
\begin{aligned}
& \Gamma_{K C}^{K} g^{C B}+\Gamma_{C K}^{B} g^{C K} \\
& =\frac{1}{2} g^{C B} g^{D E} \partial_{C} g_{D E} \\
& \quad+\frac{1}{2} g^{C K} g^{B D}\left(\partial_{K} g_{D C}+\partial_{C} g_{D K}-\partial_{D} g_{C K}+\mathbf{c}_{K C D}+\mathbf{c}_{D K C}-\mathbf{c}_{C D K}\right) \\
& =\frac{1}{2} g^{C B} g^{D E} \partial_{C} g_{D E}+g^{C K} g^{B D} \partial_{K} g_{D C}-\frac{1}{2} g^{C K} g^{B D} \partial_{D} g_{C K} \\
& =g^{C K} g^{B D} \partial_{K} g_{D C}=-\partial_{K} g^{B K},
\end{aligned}
$$

where all the terms involving structure constants vanish by (iii), and in the last line we again use the formula for the derivative of the matrix inverse. We have thus proven (24) and obtained the following equality:

$$
\delta \text { ric }_{A B} g^{A B}=\frac{1}{\sqrt{|g|}} \partial_{C}\left[\left(g^{A B} \delta \Gamma_{A B}^{C}-g^{C B} \delta \Gamma_{B A}^{A}\right) \sqrt{|g|}\right] .
$$

But this equality immediately implies (20) because one can replace the index $C$ on the right-hand side of (25) with the index $\mu$ (this can be done because every inner derivation yields zero when acting on any element of the center).

Having proven (20), we can omit the second integral in (19) and use the action principle to obtain the following generalized Einstein equations:

$$
\operatorname{ric}_{A B}-\frac{1}{2} r g_{A B}=0
$$


which can be immediately reduced to

$$
\operatorname{ric}_{A B}=0 \text {. }
$$

Therefore, one can say that endowing the differential algebra $\left(\mathcal{A}_{n}\right.$, Der $\left.\mathcal{A}_{n}\right)$ with the Einstein-Hilbert action given by (18) grants it the structure of a noncommutative Einstein algebra $[24,25] 10$.

Interestingly, these generalized Einstein equations, after being projected onto space-time $M$, have a richer form than the standard Einstein equations. This is because of the extra terms coming from additional components of the metric. These extra terms could be interpreted as an "Ndimensional matter-energy" induced by the generalized (vacuum) Einstein equations (similarly as in the Kaluza-Klein-type theories [17, 18, 19, 20]). We shall demonstrate this effect by considering an example of a simple block diagonal metric.

\section{Model with a simple metric.}

From now on, we consider a metric $\mathcal{G}$ that does not "mix" the horizontal derivations with the inner derivations

$$
\forall \bar{X} \in \operatorname{Hor} \mathcal{A}_{n} \quad \forall \operatorname{ad}_{a} \in \operatorname{Inn} \mathcal{A}_{n} \quad \mathcal{G}\left(\bar{X}, \operatorname{ad}_{a}\right)=\mathcal{G}\left(\operatorname{ad}_{a}, \bar{X}\right)=0 .
$$

Therefore, the metric matrix $\left(g_{A B}\right)$ assumes the following block diagonal form

$$
g_{A B}=\left[\begin{array}{cc}
g_{\mu \nu} & 0 \\
0 & g_{\{A\}\{B\}}
\end{array}\right] .
$$

We recall that $\left(g_{\mu \nu}\right)$ is an " $N$ by $N$ " matrix $(N=\operatorname{dim} M)$ and $\left(g_{\{A\}\{B\}}\right)$ is a " $\left(n^{2}-1\right)$ by $\left(n^{2}-1\right) "(n=|G|)$ matrix. Of course, the inverse metric matrix $\left(g^{A B}\right)$ is also block diagonal.

Let us emphasize that by restricting our attention to the metric matrices of the form (27) one does not erase the noncommutativity of the model as the structure constants $\mathbf{c}_{A B}{ }^{C}$ are still given by formulae (2 5 ). On the other hand, some more of the completely covariant structure constants are now vanishing. In fact, formulae (6 8 ) imply that

\footnotetext{
${ }^{10}$ Technically, one has yet to impose the condition that the metric matrix is lorentzian and that the Levi-Civita connection $\nabla$ can be used to define the covariant derivative. Both these requirements can be here easily met.
} 
(v) Only the completely covariant structure constants of the form $\mathbf{c}_{\{A\}\{B\}\{C\}}$ are nonzero.

With properties (i-iv) (listed in Section 4) and property (v), we are ready to compute the Christoffel symbols and the curvature tensor components associated with the block diagonal metric matrix (27).

By using general formula (10) for the components $\Gamma_{A B C}$ of the Levi-Civita preconnection one obtains

$$
\begin{aligned}
\Gamma_{\lambda \mu \nu} & =\frac{1}{2}\left(\partial_{\nu} g_{\lambda \mu}+\partial_{\mu} g_{\lambda \nu}-\partial_{\lambda} g_{\mu \nu}\right)=\widetilde{\Gamma}_{\lambda \mu \nu}, \\
\Gamma_{\{A\} \mu \nu} & =0, \quad \Gamma_{\lambda\{B\} \nu}=0, \quad \Gamma_{\lambda \mu\{C\}}=0, \\
\Gamma_{\lambda\{B\}\{C\}} & =-\frac{1}{2} \partial_{\lambda} g_{\{B\}\{C\}}, \\
\Gamma_{\{A\} \mu\{C\}} & =\frac{1}{2} \partial_{\mu} g_{\{A\}\{C\}}, \\
\Gamma_{\{A\}\{B\} \nu} & =\frac{1}{2} \partial_{\nu} g_{\{A\}\{B\}}, \\
\Gamma_{\{A\}\{B\}\{C\}} & =\frac{1}{2}\left(\mathbf{c}_{\{C\}\{B\}\{A\}}+\mathbf{c}_{\{A\}\{C\}\{B\}}+\mathbf{c}_{\{A\}\{B\}\{C\}}\right),
\end{aligned}
$$

where from now on a tilde ( $)$ above an object will mean that the object is "classical", i.e. it is obtained and used according to the standard, pseudoRiemannian-geometric formulae that employ only the "horizontal" part of the metric matrix $\left(g_{\mu \nu}\right)$.

By raising the first index in (28 33) one immediately obtains the components $\Gamma_{B C}^{A}$ of the Levi-Civita connection

$$
\begin{aligned}
\Gamma_{\mu \nu}^{\kappa} & =\frac{1}{2} g^{\kappa \lambda}\left(\partial_{\nu} g_{\lambda \mu}+\partial_{\mu} g_{\lambda \nu}-\partial_{\lambda} g_{\mu \nu}\right)=\widetilde{\Gamma}_{\mu \nu}^{\kappa}, \\
\Gamma_{\mu \nu}^{\{A\}} & =0, \quad \Gamma_{\{B\} \nu}^{\kappa}=0, \quad \Gamma_{\mu\{C\}}^{\kappa}=0, \\
\Gamma_{\{B\}\{C\}}^{\kappa} & =-\frac{1}{2} g^{\kappa \lambda} \partial_{\lambda} g_{\{B\}\{C\}}, \\
\Gamma_{\mu\{C\}}^{\{A\}} & =\frac{1}{2} g^{\{A\}\{D\}} \partial_{\mu} g_{\{D\}\{C\}}, \\
\Gamma_{\{B\} \nu}^{\{A\}} & =\frac{1}{2} g^{\{A\}\{D\}} \partial_{\nu} g_{\{D\}\{B\}}, \\
\Gamma_{\{B\}\{C\}}^{\{A\}} & =\frac{1}{2} g^{\{A\}\{D\}}\left(\mathbf{c}_{\{C\}\{B\}\{D\}}+\mathbf{c}_{\{D\}\{C\}\{B\}}+\mathbf{c}_{\{D\}\{B\}\{C\}}\right) .
\end{aligned}
$$

Thanks to the block-diagonality of the metric matrix, these formulae are still relatively simple.

Let us take a closer look at (34) and (35). By (11) they imply that

$$
\forall \bar{X}, \bar{Y} \in \operatorname{Hor} \mathcal{A}_{n} \quad \nabla_{\bar{X}} \bar{Y}=\overline{\widetilde{\nabla}_{X} Y}
$$


In other words, for the metrics whose matrices are of the form (27), the Levi-Civita connection acts on horizontal derivations in exactly the same way as does its classical counterpart. One can thus say that $\nabla$ is a (nontrivial) extension of $\widetilde{\nabla}$.

For comparison as well as for further use, let us introduce the trivial extension of the classical Levi-Civita connection $\widehat{\nabla}: V \times V \rightarrow V$ via

$$
\forall \bar{X}, \bar{Y} \in \operatorname{Hor} \mathcal{A}_{n} \quad \forall \operatorname{ad}_{a}, \operatorname{ad}_{b} \in \operatorname{Inn} \mathcal{A}_{n} \quad \widehat{\nabla}_{\bar{X} \operatorname{ad}_{a}}\left(\bar{Y}+\operatorname{ad}_{b}\right):=\overline{\widetilde{\nabla}_{X} Y} .
$$

It is indeed a well-defined connection (i.e. it has the properties $1^{\circ}-4^{\circ}$ listed in Section 3.), however it is neither torsion-free nor metric-compatible (i.e. it fails to satisfy $5^{\circ}$ and $6^{\circ}$ ).

We now move to computing the components of the Riemann tensor. Either directly from (40) or through computations employing (14) one obtains

$$
\begin{aligned}
R_{\sigma \mu \nu}^{\rho} & =\partial_{\mu} \Gamma_{\sigma \nu}^{\rho}-\partial_{\nu} \Gamma_{\sigma \mu}^{\rho}+\Gamma_{\sigma \nu}^{\kappa} \Gamma_{\kappa \mu}^{\rho}-\Gamma_{\sigma \mu}^{\kappa} \Gamma^{\rho}{ }_{\kappa \nu}=\widetilde{R}_{\sigma \mu \nu}^{\rho}, \\
R_{\sigma \mu \nu}^{\{C\}} & =0, \quad R_{\{D\} \mu \nu}^{\rho}=0, \quad R_{\sigma\{A\} \nu}^{\rho}=0, \quad R_{\sigma \mu\{B\}}^{\rho}=0 .
\end{aligned}
$$

By using (14), one also obtains that

$$
\begin{aligned}
R_{\sigma\{A\} \nu}^{\{C\}}= & -\frac{1}{2} g^{\{C\}\{E\}} \partial_{\nu} \partial_{\sigma} g_{\{E\}\{A\}}+\frac{1}{2} \Gamma_{\sigma \nu}^{\lambda} g^{\{C\}\{E\}} \partial_{\lambda} g_{\{E\}\{A\}} \\
& -\frac{1}{4} \partial_{\nu} g^{\{C\}\{E\}} \partial_{\sigma} g_{\{E\}\{A\}} \\
= & -\frac{1}{2} g^{\{C\}\{E\}} \widehat{\nabla}_{\nu} \partial_{\sigma} g_{\{E\}\{A\}}-\frac{1}{4} \partial_{\nu} g^{\{C\}\{E\}} \partial_{\sigma} g_{\{E\}\{A\}},
\end{aligned}
$$

where $\widehat{\nabla}$ denotes the covariant derivative arising from the trivial extension of the classical Levi-Civita connection (41). For practical purposes it is important to notice that when acting on tensors written in the index notation, $\widehat{\nabla}_{\nu}$ takes into account only their lowercase Greek indices. That is why in (44) one could use the fact that $\widehat{\nabla}_{\nu} \partial_{\sigma} g_{\{E\}\{A\}}=\partial_{\nu} \partial_{\sigma} g_{\{E\}\{A\}}-\Gamma_{\sigma \nu}^{\lambda} \partial_{\lambda} g_{\{E\}\{A\}}$.

All other components of the Riemann tensor that involve exactly two lowercase Greek indices can be obtained from $R_{\sigma\{A\} \nu}^{\{C\}}$ with the help of symmetries (15). For example,

$$
\begin{aligned}
& R_{\{A\} \sigma\{B\}}^{\rho}=g^{\rho \lambda} R_{\lambda\{A\} \sigma\{B\}}=g^{\rho \lambda} R_{\{A\} \lambda\{B\} \sigma}=g^{\rho \lambda} g_{\{A\}\{C\}} R_{\lambda\{B\} \sigma}^{\{C\}} \\
& =-g^{\rho \lambda} g_{\{A\}\{C\}}\left(\frac{1}{2} g^{\{C\}\{D\}} \widehat{\nabla}_{\sigma} \partial_{\lambda} g_{\{D\}\{B\}}+\frac{1}{4} \partial_{\sigma} g^{\{C\}\{D\}} \partial_{\lambda} g_{\{D\}\{B\}}\right) \\
& =g^{\rho \lambda}\left(-\frac{1}{2} \widehat{\nabla}_{\sigma} \partial_{\lambda} g_{\{A\}\{B\}}+\frac{1}{4} g^{\{C\}\{D\}} \partial_{\sigma} g_{\{C\}\{A\}} \partial_{\lambda} g_{\{D\}\{B\}}\right) .
\end{aligned}
$$


We now have all the information needed to compute the "horizontal" components of the Ricci tensor $\mathbf{r i c}_{\mu \nu}$. Applying (42) and (44) to (16), one has

$$
\begin{aligned}
\operatorname{ric}_{\mu \nu} & =R_{\mu C \nu}^{C}=R^{\rho}{ }_{\mu \rho \nu}+R^{\{C\}}{ }_{\mu\{C\} \nu}=\widetilde{R}_{\mu \rho \nu}^{\rho}+R_{\mu\{C C, \nu}^{\{C\}} \\
& =\widetilde{\mathbf{r i c}}_{\mu \nu}-\frac{1}{2} g^{\{C\}\{D\}} \widehat{\nabla}_{\nu} \partial_{\mu} g_{\{C\}\{D\}}-\frac{1}{4} \partial_{\nu} g^{\{C\}\{D\}} \partial_{\mu} g_{\{C\}\{D\}} .
\end{aligned}
$$

This expression can be put into a more compact and symmetrical form, namely,

$$
\operatorname{ric}_{\mu \nu}=\widetilde{\mathbf{r i c}}_{\mu \nu}-\frac{1}{4}\left(\widehat{\nabla}_{\mu} \widehat{\nabla}_{\nu} \ln |\breve{g}|+g^{\{A\}\{B\}} \widehat{\nabla}_{\mu} \widehat{\nabla}_{\nu} g_{\{A\}\{B\}}\right),
$$

where we have introduced $\breve{g}:=\operatorname{det}\left(g_{\{A\}\{B\}}\right)$.

Let us now compute the "mixed" components of the Ricci tensor ric $_{\mu\{B\}}$ and $\mathbf{r i c}_{\{A\} \nu}$. Of course, by the symmetry of ric, it suffices to find the formula for one of them. One can prove that

$$
\operatorname{ric}_{\mu\{B\}}=-\frac{1}{2} \mathbf{c}_{\{B\}\{C\}\{D\}} \partial_{\mu} g^{\{C\}\{D\}}=\frac{1}{2} \partial_{\mu} \mathbf{c}_{\{B\}\{C\}\{D\}} g^{\{C\}\{D\}},
$$

where the second equality is the direct consequence of (iii).

One proves (47) by the following calculation.

$$
\begin{aligned}
\operatorname{ric}_{\mu\{B\}} & =R_{\mu C\{B\}}^{C}=R_{\mu\{C\}\{B\}}^{\{C\}}+\underbrace{R_{\mu \rho\{B\}}^{\rho}}_{=0} \\
& =\Gamma_{\mu\{B\}}^{S} \Gamma_{S\{C\}}^{\{C\}}-\Gamma_{\mu\{C\}}^{S} \Gamma_{S\{B\}}^{\{C\}}-\mathbf{c}_{\{C\}\{B\}} \Gamma_{\mu S}^{\{C\}},
\end{aligned}
$$

where we have used (14) and (43). In the last formula, one can replace, by (35), the dummy index $S$ with $\{S\}$ (in each term). Moreover, the first term now vanishes because, by (35) and (iv),

$$
\Gamma_{\{S\}\{C\}}^{\{C\}}=\Gamma_{\{S\} C}^{C}=\frac{1}{2} g^{C D} \partial_{\{S\}} g_{C D}=0 .
$$

One thus has

$$
\begin{aligned}
\operatorname{ric}_{\mu\{B\}} & =-\Gamma_{\mu\{C\}}^{\{S\}} \Gamma_{\{S\}\{B\}}^{\{C\}}-\mathbf{c}_{\{C\}\{B\}}{ }^{\{S\}} \Gamma_{\mu\{S\}}^{\{C\}} \\
& =-\Gamma_{\mu S\}}^{\{S\}}\left(\Gamma^{\{C\}}{ }_{\{S\}\{B\}}+\mathbf{c}_{\{S\}\{B\}}{ }^{\{C\}}\right)=-\Gamma_{\mu S\}}^{\{C\}} \Gamma_{\{B\}\{S\}}^{\{C\}} \\
& =-\frac{1}{2} g^{\{S\}\{T\}} \partial_{\mu} g_{\{T\}\{C\}} \Gamma_{\{B\}}^{\{C\}}{ }_{\{S\}}=\frac{1}{2} \partial_{\mu} g^{\{S\}\{T\}} g_{\{T\}\{C\}} \Gamma_{\{B\}\{S\}}^{\{C\}} \\
& =\frac{1}{2} \partial_{\mu} g^{\{S\}\{T\}} \Gamma_{\{T\}\{B\}\{S\}} \\
& =\frac{1}{4} \partial_{\mu} g^{\{S\}\{T\}}\left(\mathbf{c}_{\{S\}\{B\}\{T\}}+\mathbf{c}_{\{T\}\{S\}\{B\}}+\mathbf{c}_{\{T\}\{B\}\{S\}}\right),
\end{aligned}
$$


where in one of the terms we have interchanged the names of the indices $\{C\},\{S\}$ and then used (13), (37) and (33). To obtain (47), it only remains to employ the symmetry of $g^{\{S\}\{T\}}$ and the skew-symmetry of $\mathbf{c}_{\{T\}\{S\}\{B\}}$ in the first two indices.

Let us now move to computing the "inner" components of the Ricci tensor ric $_{\{A\}\{B\}}$. One has

$$
\operatorname{ric}_{\{A\}\{B\}}=R_{\{A\} C\{B\}}^{C}=R_{\{A\} \rho\{B\}}^{\rho}+R_{\{A\}\{C\}\{B\}}^{\{C\}} .
$$

By (45), the first term yields

$$
\begin{aligned}
R_{\{A\} \rho\{B\}}^{\rho} & =g^{\rho \lambda}\left(-\frac{1}{2} \widehat{\nabla}_{\rho} \partial_{\lambda} g_{\{A\}\{B\}}+\frac{1}{4} g^{\{C\}\{D\}} \partial_{\rho} g_{\{C\}\{A\}} \partial_{\lambda} g_{\{D\}\{B\}}\right) \\
& =-\frac{1}{8} g^{\rho \lambda}\left(3 \widehat{\Delta} g_{\{A\}\{B\}}-g_{\{A\}\{C\}} g_{\{B\}\{D\}} \widehat{\Delta} g^{\{C\}\{D\}}\right),
\end{aligned}
$$

where we have introduced the Laplace-Beltrami operator $\widehat{\Delta}:=g^{\mu \nu} \widehat{\nabla}_{\mu} \widehat{\nabla}_{\nu}$ and used the fact that

$$
\begin{aligned}
& g^{\rho \lambda} g^{\{C\}\{D\}} \partial_{\rho} g_{\{C\}\{A\}} \partial_{\lambda} g_{\{D\}\{B\}} \\
& =\frac{1}{2}\left(\widehat{\Delta} g_{\{A\}\{B\}}+g_{\{A\}\{C\}} g_{\{B\}\{D\}} \widehat{\Delta} g^{\{C\}\{D\}}\right),
\end{aligned}
$$

which in turn is equivalent to the following obvious equality:

$$
g_{\{A\}\{C\}} \widehat{\Delta}\left(g^{\{C\}\{D\}} g_{\{D\}\{B\}}\right)=0 .
$$

Computing the second term on the right-hand side of (150) gives

$$
\begin{aligned}
R^{\{C\}}{ }_{\{A\}\{C\}\{B\}} & \Gamma_{\{A\}\{B\}}^{S} \Gamma_{S\{C\}}^{\{C\}}-\Gamma_{\{A\}\{C\}}^{S} \Gamma_{S\{B\}}^{\{C\}}-\mathbf{c}_{\{C\}\{B\}} \Gamma_{\{A\} S}^{\{C\}} \\
= & \Gamma_{\{A\}\{B\}}^{\rho} \Gamma_{\rho\{C\}}^{\{C\}}-\Gamma_{\{A\}\{C\}}^{\rho} \Gamma_{\rho\{B\}}^{\{C\}}-\Gamma_{\{A\}\{C\}}^{\{S\}} \Gamma_{\{S\}\{B\}}^{\{C\}} \\
& -\mathbf{c}_{\{C\}\{B\}}{ }_{\{S\}}^{\{C\}} \Gamma_{\{A\}\{S\}}^{\{S\}} \\
= & \Gamma_{\{A\}\{B\}}^{\rho} \Gamma_{\rho\{C\}}^{\{C\}}-\Gamma_{\{A\}\{C\}}^{\rho} \Gamma_{\rho\{B\}}^{\{C\}}-\Gamma_{\{A\}\{C\}}^{\{S\}} \Gamma_{\{B\}\{S\}}^{\{C\}},
\end{aligned}
$$

where we have used (14), (48), the fact that $\mathbf{c}_{\{C\}\{B\}}{ }^{\rho}=0$ and, in the last step, we have merged the last two terms in a similar fashion as in the first two lines of (49). 
Expanding the expression above using (36), (37) and (39) yields

$$
\begin{aligned}
R^{\{C\}} & \{A\}\{C\}\{B\} \\
= & -\frac{1}{4} g^{\rho \lambda} \partial_{\lambda} g_{\{A\}\{B\}} g^{\{C\}\{D\}} \partial_{\rho} g_{\{C\}\{D\}}+\frac{1}{4} g^{\rho \lambda} \partial_{\lambda} g_{\{A\}\{C\}} g^{\{C\}\{D\}} \partial_{\rho} g_{\{B\}\{D\}} \\
& -\frac{1}{4} g^{\{S\}\{T\}} g^{\{C\}\{D\}}\left(\mathbf{c}_{\{C\}\{A\}\{T\}}+\mathbf{c}_{\{T\}\{C\}\{A\}}+\mathbf{c}_{\{T\}\{A\}\{C\}}\right) \\
& \times\left(\mathbf{c}_{\{S\}\{B\}\{D\}}+\mathbf{c}_{\{D\}\{S\}\{B\}}+\mathbf{c}_{\{D\}\{B\}\{S\}}\right) \\
= & \frac{1}{4} g^{\rho \lambda} g^{\{C\}\{D\}}\left(\partial_{\lambda} g_{\{A\}\{C\}} \partial_{\rho} g_{\{B\}\{D\}}-\partial_{\lambda} g_{\{A\}\{B\}} \partial_{\rho} g_{\{C\}\{D\}}\right) \\
& -\frac{1}{2} \mathbf{c}_{\{A\}}\{C\}\{D\} \\
& \left.\mathbf{c}_{\{B\}\{C\}\{D\}}+\mathbf{c}_{\{B\}\{D\}\{C\}}\right)+\frac{1}{4} \mathbf{c}_{\{C\}\{D\}} \mathbf{c}_{\{C\}\{D\}\{B\}},
\end{aligned}
$$

where in the last equality we have skipped some tedious, but straightforward, calculations.

Inserting (51) and (53) into (50) and simplifying thus obtained expression with the help of (52) one obtains

$$
\begin{aligned}
& \operatorname{ric}_{\{A\}\{B\}} \\
&=-\frac{1}{4}\left(\widehat{\Delta} g_{\{A\}\{B\}}-g_{\{A\}\{C\}} g_{\{B\}\{D\}} \widehat{\Delta} g^{\{C\}\{D\}}+g^{\rho \lambda} \partial_{\rho} \ln |\breve{g}| \partial_{\lambda} g_{\{A\}\{B\}}\right) \\
&-\frac{1}{2} \mathbf{c}_{\{A\}}\{C\}\{D\} \\
&\left(\mathbf{c}_{\{B\}\{C\}\{D\}}+\mathbf{c}_{\{B\}\{D\}\{C\}}\right)+\frac{1}{4} \mathbf{c}_{\{A\}}^{\{C\}\{D\}} \mathbf{c}_{\{C\}\{D\}\{B\}} .
\end{aligned}
$$

Finally, let us compute the curvature scalar $r$. Through applying (46) and (54) to (17), one obtains

$$
\begin{aligned}
r= & g^{A B} \mathbf{r i c}_{A B}=g^{\mu \nu} \mathbf{r i c}_{\mu \nu}+g^{\{A\}\{B\}} \mathbf{r i c}_{\{A\}\{B\}} \\
= & g^{\mu \nu} \widetilde{\mathbf{r i c}}_{\mu \nu}-\frac{1}{4} g^{\mu \nu}\left(\widehat{\nabla}_{\mu} \widehat{\nabla}_{\nu} \ln |\breve{g}|+g^{\{A\}\{B\}} \widehat{\nabla}_{\mu} \widetilde{\nabla}_{\nu} g_{\{A\}\{B\}}\right) \\
& -\frac{1}{4} g^{\{A\}\{B\}}\left(\widehat{\Delta} g_{\{A\}\{B\}}-g_{\{A\}\{C\}} g_{\{B\}\{D\}} \widehat{\Delta} g^{\{C\}\{D\}}+g^{\rho \lambda} \partial_{\rho} \ln |\breve{g}| \partial_{\lambda} g_{\{A\}\{B\}}\right) \\
& -\frac{1}{2} \mathbf{c}^{\{B\}\{C\}\{D\}}\left(\mathbf{c}_{\{B\}\{C\}\{D\}}+\mathbf{c}_{\{B\}\{D\}\{C\}}\right)+\frac{1}{4} \mathbf{c}^{\{C\}\{D\}\{B\}} \mathbf{c}_{\{C\}\{D\}\{B\}} \\
= & \widetilde{r}-\frac{1}{4}\left(\widehat{\Delta} \ln |\breve{g}|+g^{\{A\}\{B\}} \widehat{\Delta} g_{\{A\}\{B\}}\right) \\
& -\frac{1}{4}\left(g^{\{A\}\{B\}} \widehat{\Delta} g_{\{A\}\{B\}}-g_{\{A\}\{B\}} \widehat{\Delta} g^{\{A\}\{B\}}+g^{\rho \lambda} \partial_{\rho} \ln |\breve{g}| \partial_{\lambda} \ln |\breve{g}|\right) \\
& -\frac{1}{4} \mathbf{c}^{\{B\}\{C\}\{D\}}\left(\mathbf{c}_{\{B\}\{C\}\{D\}}+2 \mathbf{c}_{\{B\}\{D\}\{C\}}\right) .
\end{aligned}
$$

This formula can be further transformed into various equivalent forms 
through the following identities 11 :

$$
\begin{aligned}
& g^{\{A\}\{B\}} \widehat{\Delta} g_{\{A\}\{B\}}+g_{\{A\}\{B\}} \widehat{\Delta} g^{\{A\}\{B\}}=-2 \partial^{\mu} g^{\{A\}\{B\}} \partial_{\mu} g_{\{A\}\{B\}}, \\
& g^{\{A\}\{B\}} \widehat{\Delta} g_{\{A\}\{B\}}-g_{\{A\}\{B\}} \widehat{\Delta} g^{\{A\}\{B\}}=2 \widehat{\Delta} \ln |\breve{g}|, \\
& \forall f \in C^{\infty}(M) \quad \widehat{\Delta} f+\partial^{\mu} f \partial_{\mu} f=e^{-f} \widehat{\Delta} e^{f},
\end{aligned}
$$

where $\partial^{\mu}:=g^{\mu \nu} \partial_{\nu}$.

For instance, by employing (56) and (57) with $f=\frac{1}{2} \ln |\breve{g}|$, one can replace the content of the second bracket with $4 \frac{\widehat{\Delta} \sqrt{|\breve{g}|}}{\sqrt{|\breve{g}|}}$. Therefore,

$$
\begin{aligned}
r= & \widetilde{r}-\frac{\widehat{\Delta} \sqrt{|\breve{g}|}}{\sqrt{|\breve{g}|}}-\frac{1}{4}\left(\widehat{\Delta} \ln |\breve{g}|+g^{\{A\}\{B\}} \widehat{\Delta} g_{\{A\}\{B\}}\right) \\
& -\frac{1}{4}\left(\mathbf{c}^{\{B\}\{C\}\{D\}}\left(2 \mathbf{c}_{\{B\}\{D\}\{C\}}+\mathbf{c}_{\{B\}\{C\}\{D\}}\right)\right) .
\end{aligned}
$$

For the sake of convenience, let us introduce the structure scalar

$$
\mathbf{C}:=\mathbf{c}^{\{B\}\{C\}\{D\}}\left(2 \mathbf{c}_{\{B\}\{D\}\{C\}}+\mathbf{c}_{\{B\}\{C\}\{D\}}\right) .
$$

It is in general nontrivial. For instance, in the case of $G=\mathbb{Z}_{2}$ it can be expressed as

$$
\mathbf{C}=\frac{2}{\breve{g}}\left[\left(g_{\{1\}\{1\}}\right)^{2}+16 g_{\{12\}\{12\}} g_{\{21\}\{21\}}+16 g_{\{1\}\{12\}} g_{\{1\}\{21\}}-8 g_{\{1\}\{1\}} g_{\{12\}\{21\}}\right] \text {. }
$$

With the help of (58), we can write the Einstein-Hilbert action (18) more explicitly, namely,

$$
S_{E H}=\int\left[\sqrt{|\breve{g}|} \widetilde{r}-\frac{1}{4} \sqrt{|\breve{g}|}\left(\widehat{\Delta} \ln |\breve{g}|+g^{\{A\}\{B\}} \widehat{\Delta} g_{\{A\}\{B\}}+\mathbf{C}\right)\right] \sqrt{-\tilde{g}} d^{N} x,
$$

where we have expressed the determinant of the metric matrix as the product of the determinants of its blocks $|g|=-\tilde{g}|\breve{g}|$. We have also omitted the divergence term $\widehat{\Delta} \sqrt{|\breve{g}|}$.

One can regard the theory obtained in this way as an example of a scalartensor theory 12 with $n^{2}\left(n^{2}-1\right) / 2$ independent scalar fields arranged into

\footnotetext{
${ }^{11}$ Identities (55) 57) can be easily proven by direct calculations.

${ }^{12}$ See e.g. 20] and references therein.
} 
a (symmetrical and non-degenerate) matrix $\left(g_{\{A\}\{B\}}\right)$. Let us notice that the field $\sqrt{|\breve{g}|}$ plays a special role in this theory.

With the help of (46, 47, 54), we can write the generalized Einstein equations $\mathbf{r i c}_{A B}=0$ for the model with the block diagonal metric matrix in the following form:

$$
\begin{aligned}
& \widetilde{\mathbf{r i c}}_{\mu \nu}=\frac{1}{4}\left(\widehat{\nabla}_{\mu} \widehat{\nabla}_{\nu} \ln |\breve{g}|+g^{\{A\}\{B\}} \widehat{\nabla}_{\mu} \widehat{\nabla}_{\nu} g_{\{A\}\{B\}}\right) \\
& \mathbf{c}_{\{B\}}\{C\}\{D\} \\
& \widehat{\Delta}_{\mu} g_{\{C\}\{D\}}=0, \\
& \quad=-2 \mathbf{c}_{\{A\}}{ }_{\{C\}\{D\}}\left(\mathbf{c}_{\{B\}\{C\}\{D\}}+\mathbf{c}_{\{B\}\{D\}\{C\}}\right)+\mathbf{c}_{\{C\}\{D\}}^{\{A\}} \mathbf{c}_{\{C\}\{D\}\{B\}} .
\end{aligned}
$$

Equation (59) is a projection of the generalized Einstein equations onto the $N$-dimensional space-time $M$. Because it implies that

$$
\widetilde{r}=\frac{1}{4}\left(\widetilde{\Delta} \ln |\breve{g}|+g^{\{A\}\{B\}} \widetilde{\Delta} g_{\{A\}\{B\}}\right),
$$

we can equivalently write (59) in the form of the standard Einstein equations with a certain nonzero energy-momentum tensor

$$
\begin{aligned}
\widetilde{G}_{\mu \nu}=\frac{1}{4} & {\left[\left(\widehat{\nabla}_{\mu} \widehat{\nabla}_{\nu}-\frac{1}{2} g_{\mu \nu} \widehat{\Delta}\right) \ln |\breve{g}|\right.} \\
& +g^{\{A\}\{B\}}\left(\widehat{\nabla}_{\mu} \widehat{\nabla}_{\nu}-\frac{1}{2} g_{\mu \nu} \widehat{\Delta}\right) g_{\{A\}\{B\}]},
\end{aligned}
$$

where $\widetilde{G}_{\mu \nu}:=\widetilde{\mathbf{r i c}}_{\mu \nu}-\frac{1}{2} g_{\mu \nu} \widetilde{r}$ is the "classical" Einstein tensor.

The appearance of a nonzero energy-momentum tensor can be regarded as a realization of the "matter-out-of-geometry" mechanism [5] or, more precisely, of the "scalar-fields-out-of-noncommutative-geometry" mechanism. Equations (60) and (61) should then be regarded as the equations of state of the scalar fields under consideration.

The continuity equation satisfied by this energy-momentum tensor can be written in the form

$$
\widetilde{\mathbf{r i c}}_{\mu \nu} \partial^{\mu} \ln |\breve{g}|+\partial_{\nu} \widetilde{r}+\frac{1}{2} \partial^{\mu} g^{\{A\}\{B\}}\left(\widehat{\nabla}_{\mu} \widehat{\nabla}_{\nu}-g_{\mu \nu} \widehat{\Delta}\right) g_{\{A\}\{B\}}=0
$$

To prove (64), let us act on both sides of (63) with the operator $4 \widehat{\nabla}^{\mu}$. Since $\widetilde{G}$ is a divergence-free tensor, the left-hand side becomes zero and one 
obtains that

$$
0=\widehat{\nabla}^{\mu}\left[\left(\widehat{\nabla}_{\mu} \widehat{\nabla}_{\nu}-\frac{1}{2} g_{\mu \nu} \widehat{\Delta}\right) \ln |\breve{g}|+g^{\{A\}\{B\}}\left(\widehat{\nabla}_{\mu} \widehat{\nabla}_{\nu}-\frac{1}{2} g_{\mu \nu} \widehat{\Delta}\right) g_{\{A\}\{B\}}\right] .
$$

By expanding the right-hand side of the equation above, one readily obtains (64), because

$$
\begin{aligned}
\widehat{\nabla}^{\mu} & {\left[\left(\widehat{\nabla}_{\mu} \widehat{\nabla}_{\nu}-\frac{1}{2} g_{\mu \nu} \widehat{\Delta}\right) \ln |\breve{g}|+g^{\{A\}\{B\}}\left(\widehat{\nabla}_{\mu} \widehat{\nabla}_{\nu}-\frac{1}{2} g_{\mu \nu} \widehat{\Delta}\right) g_{\{A\}\{B\}]}\right.} \\
= & \left(\widehat{\Delta} \partial_{\nu}-\frac{1}{2} \partial_{\nu} \widehat{\Delta}\right) \ln |\breve{g}|+g^{\{A\}\{B\}}\left(\widehat{\Delta} \partial_{\nu}-\frac{1}{2} \partial_{\nu} \widehat{\Delta}\right) g_{\{A\}\{B\}} \\
& +\partial^{\mu} g^{\{A\}\{B\}}\left(\widehat{\nabla}{ }_{\mu} \partial_{\nu}-\frac{1}{2} g_{\mu \nu} \widehat{\Delta}\right) g_{\{A\}\{B\}} \\
= & {\left[\widehat{\Delta}, \partial_{\nu}\right] \ln |\breve{g}|+\frac{1}{2} \partial_{\nu} \widehat{\Delta} \ln |\breve{g}|+g^{\{A\}\{B\}}\left[\widehat{\Delta}, \partial_{\nu}\right] g_{\{A\}\{B\}}+\frac{1}{2} g^{\{A\}\{B\}} \partial_{\nu} \widehat{\Delta} g_{\{A\}\{B\}} } \\
& +\frac{1}{2} \partial_{\nu} g^{\{A\}\{B\}} \widehat{\Delta} g_{\{A\}\{B\}}+\partial^{\mu} g^{\{A\}\{B\}}\left(\widehat{\nabla}_{\mu} \widehat{\nabla}_{\nu}-g_{\mu \nu} \widehat{\Delta}\right) g_{\{A\}\{B\}} \\
= & \widetilde{\mathbf{r i c}}_{\mu \nu} \partial^{\mu} \ln |\breve{g}|+g^{\{A\}\{B\}} \widetilde{\mathbf{r i c}}_{\mu \nu} \partial^{\mu} g_{\{A\}\{B\}}+\frac{1}{2} \partial_{\nu}\left(\widetilde{\Delta} \ln |\breve{g}|+g^{\{A\}\{B\}} \widetilde{\Delta} g_{\{A\}\{B\}}\right) \\
& +\partial^{\mu} g^{\{A\}\{B\}}\left(\widehat{\nabla} \widehat{\nabla}_{\nu}-g_{\mu \nu} \widehat{\Delta}\right) g_{\{A\}\{B\}} \\
= & 2 \widetilde{\mathbf{r i c}}_{\mu \nu} \partial^{\mu} \ln |\breve{g}|+2 \partial_{\nu} \widetilde{r}+\partial^{\mu} g^{\{A\}\{B\}}\left(\widehat{\nabla} \widehat{\nabla}_{\nu}-g_{\mu \nu} \widehat{\Delta}\right) g_{\{A\}\{B\}},
\end{aligned}
$$

where we have used (62) and the identity 13

$$
\forall f \in C^{\infty}(M) \quad\left[\widehat{\Delta}, \partial_{\nu}\right] f=\widetilde{\mathbf{r i c}}_{\mu \nu} \partial^{\mu} f
$$

Let us present another remarkable equation that can be inferred from Einstein equations (59 61). Namely, by (58), (62) and the fact that $r=0$ it is true that 14

$$
\widehat{\Delta} \sqrt{|\breve{g}|}+\frac{1}{4} \mathbf{C} \sqrt{|\breve{g}|}=0
$$

Equation (66), written in this form, resembles the Klein-Gordon equation. However, the structure scalar need not be constant, therefore one should interpret it as a certain potential rather the the mass term.

\footnotetext{
${ }^{13}$ Identity (65) can be easily proven in the Riemann normal coordinates.

${ }^{14}$ Equation (66) can also be obtained from (61) through the use of the trace operation with the help of identities (55) 577).
} 
Let us finish this section by presenting two explicit Friedmann-like solutions of Einstein equations (59 61) in the simplest case when the structure group $G$ is equal to $\mathbb{Z}_{2}$. By a "Friedmann-like" solution we mean a metric, whose "horizontal" part $\left(g_{\mu \nu}\right)$ is of the Friedmann-Lemaître-RobertsonWalker form and whose remaining components depend only on the time variable 15

$$
\begin{aligned}
& g_{\mu \nu}=\left[\begin{array}{cccc}
-1 & 0 & 0 & 0 \\
0 & \frac{a^{2}(t)}{1-k r^{2}} & 0 & 0 \\
0 & 0 & a^{2}(t) r^{2} & 0 \\
0 & 0 & 0 & a^{2}(t) r^{2} \sin ^{2} \theta
\end{array}\right] \\
& g_{\{A\}\{B\}}=g_{\{A\}\{B\}}(t),
\end{aligned}
$$

where $(r, \theta, \phi)$ denote the reduced-circumference polar coordinates, $a(t)$ is the scale factor and $k \in\{-1,0,1\}$ is the curvature constant.

To further simplify calculations, we assume that the "inner" part of the metric matrix $\left(g_{\{A\}\{B\}}\right)$ has the following form:

$$
g_{\{A\}\{B\}}(t)=\left[\begin{array}{ccc}
\xi f^{2}(t) & 0 & 0 \\
0 & 0 & \eta f^{2}(t) \\
0 & \eta f^{2}(t) & 0
\end{array}\right],
$$

where $\xi, \eta$ are nonzero constants and $f$ is a time-dependent nonvanishing function.

For the metrics with the "inner" part of the form (67), the second Einstein equation (60) is satisfied automatically. The remaining two equations (59, 61) amount to the following overdetermined nonlinear system of ODEs

$$
\left\{\begin{aligned}
\frac{\ddot{a}}{a}+\frac{\ddot{f}}{f} & =0, \\
a \ddot{a} f+2 \dot{a}^{2} f+3 a \dot{a} \dot{f} & =-2 k f, \\
f \ddot{f} a+2 \dot{f}^{2} a+3 f \dot{f} \dot{a} & =\frac{1}{\eta} a .
\end{aligned}\right.
$$

together with an additional algebraical condition that $\xi=2 \eta$. Note that this system of ODEs is symmetric with respect to the interchange $(a, k) \leftrightarrow$ $\left(f,-(2 \eta)^{-1}\right)$. Bearing this in mind, we will call both functions $a$ and $f$ "scale factors".

\footnotetext{
${ }^{15}$ We adopt the system of units in which $c=G=1$.
} 
It is noteworthy that, by the third equation of (68), the function $f$ cannot be constant. Moreover, one can express the Hubble parameter $H$ in terms of $f$ and its time derivatives as

$$
H:=\frac{\dot{a}}{a}=\frac{\eta^{-1}-f \ddot{f}-2 \dot{f}^{2}}{3 f \dot{f}} .
$$

The detailed study of system (68) goes far beyond the scope of this paper and will be addressed in the future work. Here, we only find the solutions involving scale factors $a, f$ that are linear

$$
\begin{aligned}
& a(t)=a_{1}\left(t-t_{0}\right)+a_{0}, \\
& f(t)=f_{1}\left(t-t_{0}\right)+f_{0},
\end{aligned}
$$

where $a_{0}, a_{1}, f_{0}, f_{1}, t_{0}$ are constants. As such, $a$ and $f$ satisfy the first ODE in (68) trivially.

The remaining two ODEs now imply the following nonlinear system of algebraic equations:

$$
\left\{\begin{aligned}
5 a_{1}^{2} f_{1} & =-2 k f_{1}, \\
2 a_{1}^{2} f_{0}+3 a_{0} a_{1} f_{1} & =-2 k f_{0}, \\
5 f_{1}^{2} a_{1} & =\frac{1}{\eta} a_{1}, \\
2 f_{1}^{2} a_{0}+3 f_{0} f_{1} a_{1} & =\frac{1}{\eta} a_{0} .
\end{aligned}\right.
$$

One can show that this system has only two nonzero solutions, one for $k=0$ and another for $k=-1$

$$
\begin{array}{lll}
\text { for } k=0 & a(t)=a_{0}, & f(t)=\frac{1}{\sqrt{2 \eta}}\left(t-t_{0}\right), \\
\text { for } k=-1 & a(t)=\sqrt{\frac{2}{5}}\left(t-t_{0}\right), & f(t)=\frac{1}{\sqrt{5 \eta}}\left(t-t_{0}\right),
\end{array}
$$

where $\eta, a_{0}, t_{0}$ are constants. Note that $\eta$ cancels out when one substitutes the above solutions into (67), therefore without any loss of generality one can take $\eta=1$. Note also that for $t=t_{0}$ the metric matrix $\left(g_{A B}\right)$ becomes degenerate.

The first solution (69) describes the flat Minkowski space-time, although it is not a static solution since $\dot{f} \neq 0$ (and the previously mentioned degeneracy occurs at $t=t_{0}$ ).

The second solution (70) describes a hyperbolic, linearly expanding universe with the initial singularity at $t=t_{0}$. Here the Hubble parameter 
$H=\left(t-t_{0}\right)^{-1}$. The age of the universe is therefore nothing but the Hubble time $H_{0}^{-1}$, where $H_{0}$ denotes the Hubble constant 16 .

In the context of the Friedmann-like solutions, it might be interesting to see what kind of perfect fluid should be assumed in the standard Friedmann equations to obtain these particular solutions. Rephrasing the last statement, we are interested in what kind of matter-energy is in this case induced by the (noncommutative) geometry.

Of course, in the case of Minkowski solution (69) no matter-energy appears. However, in the case of hyperbolic solution (70), the "classical" Einstein tensor assumes a nontrivial form

$$
\widetilde{G}_{\mu \nu}=\frac{3}{2\left(t-t_{0}\right)^{2}} \operatorname{diag}(3,1,1,1)
$$

Therefore, the induced perfect fluid energy density $\rho$ and pressure $p$ are

$$
\rho(t)=-\frac{36 \pi}{\left(t-t_{0}\right)^{2}}, \quad p(t)=\frac{12 \pi}{\left(t-t_{0}\right)^{2}} .
$$

Note that $\rho$ is negative and therefore in this case no classical fluid matches the induced one. The equation of state of the fluid reads here $p=-\frac{1}{3} \rho$.

\section{Concluding remarks}

In the present work, we have studied a very simplified model of our approach to the unification of general relativity and quantum mechanics. The justification of this strategy is that in this simplified model many effects, which in our previous works could only be theoretically indicated, are now explicitly calculated. Although the model considered in the present work is strongly simplified, it enjoys some interesting properties that could be illuminating also for more realistic models. In the considered model, the full module of derivations Der $\mathcal{A}_{n}$ is locally free of finite rank. This fact has allowed us to construct, by using a local basis, the (smooth) geometry of the gravitational sector in a computable way. Generalized Einstein equations have been derived with the help of the action principle for a suitably defined analogue of the Einstein-Hilbert action with no matter terms. When projected onto space-time manifold, the standard Einstein equations have been recovered plus some extra term, which can be interpreted as an energy-momentum

\footnotetext{
${ }^{16}$ From the observational data $H_{0}^{-1}=(14.42 \pm 0.16) \times 10^{9}$ yr [26].
} 
tensor arising from noncommutativity, and the components of this tensor as a set of scalar fields together with their equations of state. We have been able to illustrate this "matter-out-of-geometry" mechanism by studying two explicit solutions to the generalized Einstein equations.

In the present work, we have considered the gravitational sector of our model in the case of a finite structure group. The quantum sector of this model was considered in [3, 7]. Let us take a quick look at its main features. To construct the quantum sector one considers the regular representation of the algebra $\mathcal{A}_{n}$

$$
\pi_{p}: \mathcal{A}_{n} \rightarrow \mathcal{B}\left(\mathcal{H}_{p}\right)
$$

in the collection of Hilbert spaces $\mathcal{H}_{p}:=L^{2}\left(\Gamma^{p}\right)$. This representation establishes an isomorphism between $\mathcal{A}_{n}$ and $\mathcal{M}_{0}:=\pi\left(\mathcal{A}_{n}\right)$ where, for any $a \in \mathcal{A}_{n}, \pi(a):=\left(\pi_{p}(a)\right)_{p \in M \times G}$. This isomorphism allows us to transfer geometry of the gravitational sector to the quantum sector. It is remarkable that the geometry, as transferred to $\mathcal{M}_{0}$, has a strong probabilistic flavor. Indeed, the operators $\pi(a)$ are random operators [9]. To obtain the "full" quantum sector, we complete $\mathcal{M}_{0}$ to a von Neumann algebra $\mathcal{M}:=\mathcal{M}_{0}^{\prime \prime}$, where the operators $\left(\pi_{p}(a)\right)_{p \in M \times G}$ act in the Hilbert space given by the direct integral [3]

$$
\mathcal{H}:=\int_{p \in M \times G} \mathcal{H}_{p} d \mu(p)
$$

The isomorphism $\mathcal{A}_{n} \cong \mathcal{M}_{0}$ does not extend to $\mathcal{M}$. This shows that geometric methods have a limited range in the quantum sector. This sector will be studied, in a more detailed way, in a forthcoming work.

However, one should remember that the model with a finite structure group is to be regarded as a step towards constructing more physically realistic models.

\section{References}

[1] M. Heller, W. Sasin, D. Lambert, Groupoid Approach to Noncommutative Quantization of Gravity, J. Math. Phys. 38(11), 5840-5853 (1997). 
[2] M. Heller, W. Sasin, Noncommutative Unification of General Relativity and Quantum Mechanics, Int. J. Theor. Phys. 38(6), 1619-1642 (1999).

[3] M. Heller, L. Pysiak, W. Sasin, Noncommutative Dynamics of Random Operators, Int. J. Theor. Phys. 44(6), 619-628 (2005).

[4] M. Heller, L. Pysiak and W. Sasin, Noncommutative Unification of General Relativity and Quantum Mechanics, J. Math. Phys. 46, 122501-16 (2005).

[5] M. Heller, L. Pysiak and W. Sasin, Conceptual Unification of Gravity and Quanta, Int. J. Math. Phys. 46(10), 2494-2512 (2007).

[6] M. Heller, L. Pysiak, W. Sasin, Fundamental Problems in the Unification of Physics, Found. Phys. 41(5), 905-918 (2011).

[7] M. Heller, Z. Odrzygóźdź, L. Pysiak and W. Sasin, Noncommutative Unification of General Relativity and Quantum Mechanics. A Finite Model, Gen. Rel. Grav. 36(1), 111-126 (2004).

[8] M. Heller, Z. Odrzygóźdź, L. Pysiak and W. Sasin, Observables in a Noncommutative Approach to the Unification of Quanta and Gravity: A Finite Model, Gen. Rel. Grav. 37(3), 541-555 (2005).

[9] A. Connes, Noncommutative Geometry, Academic Press, New York, 1994.

[10] J. Madore, An Introduction to Noncommutative Differential Geometry and Its Physical Applications, 2nd ed. Cambridge University Press, Cambridge, 1999 .

[11] J.M. Gracia-Bondía, J.C. Várilly, H. Figueroa, Elements of noncommutative geometry, Birkhäuser, Boston, 2001.

[12] M. Dubois-Violette, Dérivations et calcul différentiel non-commutatif, C.R. Acad. Sci. Paris 307(1), 403-408 (1988).

[13] M. Dubois-Violette, R. Kerner and J. Madore, Noncommutative differential geometry of matrix algebras, J. Math. Phys. 31, 316-322 (1990).

[14] A.E.F. Djemai, Introduction to Dubois-Violette's Noncommutative Differential Geometry, Int. J. Theor. Phys. 34(6), 801-887 (1995). 
[15] M. Heller, Einstein Algebras and General Relativity, Int. J. Theor. Phys. 31(2), 277-288 (1992).

[16] M. Heller, W. Sasin, Structured Spaces and Their Application to Relativistic Physics, J. Math. Phys. 36(7), 3644-3662 (1995).

[17] H.C. Lee (Editor), An introduction to Kaluza-Klein theories, proc. Chalk River workshop on Kaluza-Klein theories, World Scientific, Singapore. 1984 .

[18] T. Appelquist, A. Chodos, and P.G.O. Freund (Editors), Modern Kaluza-Klein theories, Addison-Wesley, Menlo Park. 1987.

[19] J.M. Overduin, and P.S. Wesson, Kaluza-Klein Gravity, Phys. Rep. 283(5-6), 303 Ü378 (1997).

[20] T. Clifton, P.G. Ferreira, A. Padilla, C. Skordis, Modified Gravity and Cosmology, Phys. Rep. 513(1), 1-189 (2012).

[21] S. Azam, Derivations of tensor products algebras, Comm. Alg. 36(3), 905-227 (2008).

[22] G.N. Parfionov and R.R. Zapatrin, Pointless Spaces in General Relativity, Int. J. Theor. Phys. 34(5), 717-731 (1995).

[23] W. Sasin and M. Heller, Non-Commutative Differential Geometry, Acta Cosmologica 21(2), 235-245 (1995).

[24] R. Geroch, Einstein Algebras, Comm. Math. Phys. 26(4), 271-275 (1972).

[25] M. Heller, Commutative and Non-Commutative Einstein algebras, Acta Cosmologica 21(2), 111-130 (1995).

[26] Planck Collaboration, Planck 2013 results. I. Overview of products and scientific results, arXiv:1303.5062. 